\title{
Multi-player Multi-issue Negotiation with Complete Information
}

\author{
Mengxiao $\mathrm{Wu}$ \\ CWI, Centre for Mathematics \\ and Computer Science \\ Amsterdam, The Netherlands \\ wu@cwi.nl \\ Chetan Yadati \\ Delft University of Technology \\ Delft, The Netherlands \\ c.yadati@tudelft.nl
}

\author{
Mathijs de Weerdt \\ Delft University of Technology \\ Delft, The Netherlands \\ M.M.deWeerdt@tudelft.nl \\ Yingqian Zhang \\ Delft University of Technology \\ Delft, The Netherlands \\ Yingqian.Zhang@tudelft.nl
}

\author{
Han La Poutré \\ $\mathrm{CWI}$, Centre for Mathematics \\ and Computer Science \\ Amsterdam, The Netherlands \\ hlp@cwi.nl
}

\author{
Cees Witteveen \\ Delft University of Technology \\ Delft, The Netherlands \\ c.Witteveen@tudelft.nl
}

\begin{abstract}
This paper presents a multi-player multi-issue negotiation model to solve a resource allocation problem. We design a multilateral negotiation protocol, by which rational players bid sequentially in consecutive rounds till a deadline. Every player's bid is a combination of all resource allocations for himself. In this framework, we perform a thorough theoretical analysis of the negotiation with complete information, which is a preliminary for the more complex incomplete information case. We show that, under a complete information setting, we can derive the negotiation strategies that form a subgame perfect equilibrium outcome. We also show that when a discount factor exists, an agreement will be reached immediately at the end of the first negotiation round. By making trade-offs between issues, the utility that every player gets in the equilibrium outcome is maximized and the solution is Pareto optimal.
\end{abstract}

\section{INTRODUCTION}

With the rapid development of multi-agent systems, automated negotiation has been widely used to solve coordination and cooperation problems in complex systems. In this paper, we propose a solution when multiple players allocate multiple resources amongst themselves through negotiation. In contrast to most previous work on two-player multi-issue negotiation [6] or multi-player single-issue negotiation $[2,9$, 16], the negotiation model presented in this work is a multiplayer multi-issue strategic negotiation model. It is also different from the model of multiple bilateral negotiation between more than two players [8]; it is a multilateral negotiation that always includes all players in one negotiation. Thus, the negotiation model proposed by us is more general and applies to multi-issue negotiation between more than

Permission to make digital or hard copies of all or part of this work for personal or classroom use is granted without fee provided that copies are not made or distributed for profit or commercial advantage and that copies bear this notice and the full citation on the first page. To copy otherwise, to republish, to post on servers or to redistribute to lists, requires prior specific permission and/or a fee.

Copyright 200X ACM X-XXXXX-XX-X/XX/XX ...\$5.00. two players in the real world.

We design a negotiation protocol when each player bids a combination of desirable allocations only for himself not for all players. Compared to Rubinstein's alternating-offer bargaining [14], in which one player's proposal includes allocations for all players, this model applies to many real negotiation scenarios and the equilibrium solution applies to those scenarios directly. Fatima et al [6] study different approaches to multi-issue negotiation and conclude that the package approach, when all issues are bundled and negotiated concurrently, is the optimal way for multi-issue negotiation. In our work, we extend this concurrent multi-issue negotiation model from two players to multiple players, in a model where we allow all players to bid combinations for all resource allocations. The model presented in this paper tackles several problems introduced by multi-player negotiation. It shows the bidding order problem in the negotiation between more than two players and provides a simple way to solve it. The change from bidding allocations for all players to bidding allocations for each player himself increases the opportunities of learning under incomplete information environments. Although we just analyze the negotiation with complete information in this paper, the proposed model is a fundamental result of automated negotiation studied. This paper is an important step towards the incomplete information case and provides a benchmark for multi-player multiissue negotiation.

We briefly describe the negotiation model here and present the full details in the next sections. We study solutions with $n \geq 2$ players to allocate $m \geq 2$ resources amongst themselves through negotiation, which takes place round by round under a time constraint, a negotiation deadline. All players have to reach an allocation agreement, otherwise no resource will be allocated. Different from the two-player negotiation that a player's allocation determines his opponent's allocation indirectly, in the $n$-player negotiation, even if one player's allocation is determined separately, the rest of the players still need to negotiate allocations of the issues left. Because every player's focus is his own desirable allocation, instead of one player's proposal for all players' allocations [2], we let $n$ players make bids/responses sequentially in each round and let every player's bid be a combination of all resource allocations only for himself. By making 
trade-offs between allocations of different resources, the outcome utility that every player gets can be maximized while the utilities that his opponents get are kept, so it is easier to reach an allocation agreement than negotiating resources separately. We set the negotiation under a complete information environment, in which all information is common knowledge, and develop equilibrium strategies of the players. The outcome formed by those strategies is a subgame perfect equilibrium (SPE) [15]. Given another time constraint, a discount factor that decreasing the utilities of bids during the negotiation, an agreement can be reached immediately at the end of the first round. Further, the solution is Pareto optimal when each player benevolently select the bid amongst multiple bids that would introduce the same utility to him.

The rest of the paper is organized as follows. Section 2 describes the negotiation model including the problem model, the negotiation protocol and utility functions. Section 3 proposes the equilibrium strategies formally and proves several properties of the outcome. A simple example of the equilibrium strategies is also given. Section 4 gives a brief summary of related work. And finally, Section 5 presents the conclusions and future work.

\section{THE NEGOTIATION MODEL}

Suppose a complex task requires a finite set of $n \geq 2$ players to perform, given a finite set of $m \geq 2$ divisible resources. The task is divided into $n$ subtasks, each of which is allocated to one player. We assume every player needs a combination of all types of resources to perform his subtask. Hence, a player's allocation is a combination of every resource allocated to him. Every player's allocation can only be implemented if all players agree with it. To solve the problem of resource allocation, we propose a solution when $n$ players allocate $m$ resources amongst themselves through multilateral negotiation. For the sake of simplicity, we interpret an amount $x_{k}$ as an allocation percentage of the $k^{\text {th }}$ resource where $x_{k} \in(0,1)$ and $1 \leq k \leq m$. We denote the complete set of the total amounts of resources by a vector 1 in which every element is 1 . In the rest of this paper, we use the term issue to indicate the amount of a resource negotiated by the players.

\subsection{The Negotiation Protocol}

In this section, we impose a negotiation protocol that describes how players can act and interact during the negotiation. We let the negotiation take place round by round $r \in \mathbb{N}$, in which the players can take actions. There are two common time constraints, a negotiation deadline $\gamma \in \mathbb{N}$ and a constant discount factor $\delta \in(0,1)$. If the players cannot reach an allocation agreement on all issues in any round $r \leq \gamma$, the negotiation fails and all players get nothing. We let disagreement denote this outcome, which is the worst outcome of this negotiation. Given an allocation agreement at a subsequent moment, the utility that a player gets is decreased by the discount $\delta$.

We let $n$ players take actions sequentially in consecutive rounds till the deadline $\gamma$. Different from Rubinstein's alternating-offer bargaining [14], in each round $r \leq \gamma$, we let each player bid one desirable combination of the $m$ issues for himself sequentially, given the bidding order of the current round $r$. In this work, the negotiation protocol requires the bidding orders of all rounds to be pre-specified and fixed during the whole negotiation, but the way to generate the bidding orders can be various. A given player is represented by a different bidder in each of the rounds, provided that those bidders all share the same preference and information of the original player. For instance, in the case of three players 1,2 and 3 , the bidding orders can be $\langle(1,2,3),(2,3,1),(3,1,2), \ldots\rangle$, in which player 1 is represented by the first bidder, the third bidder and the second bidder in rounds 1,2 and 3 respectively. We let bidder $i \in \mathbb{N}$ represent the $i^{t h}$ bidder in a round where $1 \leq i \leq n$ and let $N$ denote the set of bidders $\{1, \ldots, n\}$ of a round.

When it is a bidder's turn to bid in round $r \leq \gamma$, given the bids of the previous bidders in the current round, the bidder can either accept those previous bids and make his own bid, or reject those bids and the chance of bidding. At the beginning of every round $r$, the issues available for bidders to bid are always the complete set of issues $\mathbf{1}$. The issues cannot be bidden separately. The bidder either bids a combination of all $m$ issues or rejects to bid for any issue. Therefore, the set of all possible bids is $B=(0,1)^{m}$ and bidder $i$ 's bid is an $m$-vector $\mathbf{x}_{i} \in B$. An element ${ }^{1} x_{i, k} \in \mathbf{x}_{i}$ represents the $k^{t h}$ issue of bid $\mathbf{x}_{i}$ where $1 \leq k \leq m$. We let reject denote the action of rejection. Therefore, the set of all possible actions of every bidder is $A=B \cup\{$ reject $\}$. We let $a_{i} \in A$ denote bidder $i$ 's action in a round and let $a \in A^{n}$ denote an action profile chosen by $n$ bidders in the round. We let $\mathbf{x} \in B^{n}$ denote a bid profile and define an agreement to be:

$$
\mathbf{x}=\left(\mathbf{x}_{1}, \ldots, \mathbf{x}_{n}\right) \text { subject to } \forall k \in\{1, \ldots, m\} \sum_{i=1}^{n} x_{i, k} \leq 1
$$

Note that for the notational simplicity, we eliminate the time indexes of the notations of bidder $i$, bid $\mathbf{x}_{i}$, the bid profile $\mathbf{x}$, action $a_{i}$ and the action profile $a$. In the rest of this work, those notations are always bounded to the round that the negotiation is taking place, unless specified otherwise.

Further, we make the following assumptions about the players and the negotiation.

- Unanimity: only a unanimous agreement can be accepted and then be implemented.

- Rationality: every player will act in order to maximize his own utility.

- Patience: all players are patient enough to stay in the negotiation till the deadline $\gamma$, if no agreement has been reached yet.

- Benevolence: when a player can choose between multiple outcomes which are indifferent to him but not to his opponents, he will choose the one that is best for his opponents as far as he knows.

In this work, we assume the negotiation takes place under a complete information setting. The time constraints, the above assumptions and the preferences of players are all common knowledge. Given the definitions and assumptions above, we propose the negotiation protocol.

- In each negotiation round $r \leq \gamma$, from the first bidder to the last bidder, every bidder $i \in N$ takes an action $a_{i} \in A$ sequentially.

${ }^{1}$ In this paper, we also use $\epsilon$ to represent the relation that an element belongs to a vector. 
- In round $r<\gamma$, given all previous bids $\left(\mathbf{x}_{1}, \ldots, \mathbf{x}_{i-1}\right)$, bidder $i$ can either accept them and bid $\mathbf{x}_{i}$, or reject them. If bidder $i$ bids $\mathbf{x}_{i}$, then it is bidder $i+1$ 's turn to choose his action/response. If bidder $i$ chooses to reject the previous bids, the current round $r$ ends and the negotiation passes on to round $r+1$. Once bidder $n$ accepts all previous bids and bids $\mathbf{x}_{n}$ and the bid profile $\mathbf{x}$ satisfies the constraint $(\mathcal{C})$, an agreement is reached and the negotiation stops successfully.

- If no agreement is reached in any round $r \leq \gamma$, the negotiation stops unsuccessfully and the outcome is disagreement.

\subsection{Utility Functions}

As defined in the last section, the outcome of the negotiation is some agreement or disagreement. Each player's preference over the outcomes are represented by a utility function, which is common knowledge in this game. In the following, however, we define all functions on a player in a specific round, which is represented by a bidder. We refer to bidders representing the same player in different rounds the same information. (Recall that the mapping of any player and the bidders representing him is specified by the bidding orders.)

Because of the discount factor $\delta$, an agreement reached in different rounds introduces different utilities to the players. Hence, the utility depends on not only the action profile but the time as well. We define the utility function $u_{i}: A^{n} \times \mathbb{N} \rightarrow$ $\mathbb{R}$, where $u_{i}(a, r)$ represents the utility that bidder $i \in N$ would get in round $r \in \mathbb{N}$, if the bidders all chose their actions as specified in $a \in A^{n}$. Note that disagreement only happens at the end of the negotiation deadline $\gamma$, when (i) an action of reject exists in the action profile $a$, or (ii) $a$ is a bid profile $\mathbf{x}$ but does not satisfy the constraint $(\mathcal{C})$. An agreement may be reached in any round $r \leq \gamma$, when $a$ is a bid profile $\mathbf{x}$ and the constraint $(\mathcal{C})$ is satisfied. In this situation, the utility of $\mathbf{x}$ for bidder $i$ only depends on his bid $\mathbf{x}_{i} \in \mathbf{x}$; we define a general valuation function $v_{i}$ : $B \rightarrow \mathbb{R}$ to calculate the value. In this work, we assume the valuation function to be a monotonically increasing function of any element $x_{i, k} \in \mathbf{x}_{i}(1 \leq k \leq m)$. Formally, the utility function is given by:

$$
u_{i}(a, r)=\left\{\begin{aligned}
0 & \text { if } r=\gamma \text { and reject } \in a \\
0 & \text { if } r=\gamma, a=\mathbf{x} \text { and not }(\mathcal{C}) \\
\delta^{r-1} \cdot v_{i}\left(\mathbf{x}_{i}\right) & \text { if } r \leq \gamma, a=\mathbf{x} \text { and }(\mathcal{C})
\end{aligned}\right.
$$

where $\mathbf{x}_{i} \in a$.

Note that the range of $v_{i}\left(\mathbf{x}_{i}\right)$ is $\mathbb{R}$ but not $\mathbb{R}^{+}$, which means the value of a combination of allocations for bidder $i$ can be negative, so the utility of an action profile $a$ for bidder $i$ can be negative, if $a$ actually happens. However, because the rational bidders prefer disagreement to the allocations with a negative utility, that actions profile $a$ cannot really form an outcome of this game and disagreement is the worst outcome of this game. We explain this point in order to distinguish the problem in this work from the typical problem of a cake partition. To reach an agreement, in which his opponents accept allocations with zero utilities at the end of the game, the bidding player still has to leave a minimum amount of resources to them but not nothing. This point makes the problem setting in this work relevant to more scenarios in the real world.

\section{THE NEGOTIATION STRATEGIES}

In this section, we investigate the equilibrium strategies of the players of the game and use the notion of subgame perfect equilibrium [15], which induces a Nash equilibrium in every subgame (round), to examine the solution formed by those equilibrium strategies.

\subsection{Description of Strategies}

We analyze the equilibrium strategies to specify the optimal action of every bidder $i \in N$ in any round $r \leq \gamma$, when it is his turn to bid, given the previous bids in round $r$. Bidder $i$ ' optimal action is to maximize the utility that he would get when the game ends. Bidder $i$ 's equilibrium strategy is to try out all possible actions in $A$ to find the one that has the maximum utility. As specified by the utility function (1), any bid's utility for bidder $i$ is not only determined by bidder $i$ 's valuation but also determined by whether his opponents accept it. Every bidder's optimal action is the one that maximizes his own utility with the consideration of his opponents' responses. Therefore, all bidders' optimal actions in a round are best responses to each other; the action profile forms a Nash equilibrium [12]. When it is bidder $i$ 's turn to bid, we let $h_{i}=\left(\mathbf{x}_{1}, \ldots, \mathbf{x}_{i-1}\right)$ denote the profile of previous bids in the current round and let $H$ denote the set of all possible profiles of bids in the game. We define the optimal action function $s_{i}: H \times \mathbb{N} \rightarrow A$, where $s_{i}\left(h_{i}, r\right)$ is bidder $i$ 's optimal action in round $r$, given previous bids $h_{i}$ in round $r$. We let $a_{i}^{*}$ denote the optimal action of bidder $i$ in the current round $r$.

We let $-i$ denote the set of all bidders other then $i$ in a round, so a combination of their actions can be represented by $a_{-i}=\times_{j \in N-i} a_{j}$. Given an action $a_{i} \in A$, bidder $i$ reasons his opponents' responses $a_{-i}$ first, and then calculates the utility of the action profile $a=\left(a_{i}, a_{-i}\right)$ for himself. The utility function (1) only gives the utilities of an action profile $a \in A^{n}$ for every player, when $a$ forms either the outcome of an agreement or disagreement in round $\gamma$. However, when $a$ cannot form an agreement in the current round $r<\gamma$ and the negotiation passes on to the next round $r+1$, the utilities of $a$ for the players have not been specified. We define the utility of an action profile $a$ for bidder $i \in N$ in round $r \leq \gamma$ to be equal to the utility that the player represented by bidder $i$ in round $r$ would get in the next round $r+1$, if $a$ cannot form an agreement in the current round $r$. Apparently, if a player chooses to reject the previous bids, the utility of reject in round $r$ just equals to the utility that the player would get in round $r+1$.

When it is bidder $i$ 's turn to bid in round $r \leq \gamma$, bidder $i$ needs to reason the utility that he would get in round $r+1$. The result is also the utility of reject in round $r$ and will be compared to the utility of any possible bid for him to determine his optimal action. To calculate the utility of any bid $\mathbf{x}_{i} \in B$, bidder $i$ needs to reason the best response of each of the remaining bidders $j>i$ in round $r$ to his possible bid $\mathbf{x}_{i}$. The best response is bidder $j$ 's optimal action derived from $(i)$ the previous bids $\left(\mathbf{x}_{1}, \ldots, \mathbf{x}_{i-1}\right),(i i)$ the possible bid $\mathbf{x}_{i}$ of bidder $i$ and (iii) the reasoned optimal action of bidder $j^{\prime}$ where $i<j^{\prime}<j$. The reasoning also requires the information of the utilities that all players would get in the next round $r+1$. Eventually, bidder $i$ in round $r$ does the reasoning from bidder $i+1$ to bidder $n$ in round $r$, continues it from the first bidder to the last bidder in round $r+1$, and lasts it till the last bidder in round $\gamma$. It is 
a recursive procedure with a base case that all players will get zero utilities after round $\gamma$, if no agreement has been reached.

\subsection{Formal Definition of Strategies}

Given the description above, we formally define the optimal function and present the negotiation strategies. We develop some notations first. As the presentation of the strategies is concerned with the consecutive rounds, we let $r$ and $r+1$ denote the current round and the next round respectively. We use a letter and the letter with a tilde to denote a bidder of round $r$ and a bidder of round $r+1$ respectively, which represent the same original player. For instance, bidders $i$ and $\tilde{\imath}$ denote the $i^{t h}$ and $\tilde{i}^{t h}$ bidders in rounds $r$ and $r+1$ respectively; they have the same utility function as they represent the same player.

Formally, given the previous bids $h_{i}=\left(\mathbf{x}_{1}, \ldots, \mathbf{x}_{i-1}\right)$, the optimal action function is defined by:

$$
s_{i}\left(h_{i}, r\right) \in \underset{a_{i} \in A}{\operatorname{argmax}} w_{i}\left(a_{i}, h_{i}, r\right)
$$

where

$$
w_{i}\left(a_{i}, h_{i}, r\right)=\left\{\begin{aligned}
0 & \text { if } r>\gamma \\
u_{i}(\mathbf{x}, r) & \text { if } r \leq \gamma, a=\mathbf{x} \text { and }(\mathcal{C}) \\
w_{\tilde{\imath}}\left(a_{\tilde{\imath}}^{*}, h_{\tilde{\imath}}, r+1\right) & \text { otherwise }
\end{aligned}\right.
$$

where

$$
\begin{gathered}
a=\left(h_{i}, a_{i}, a_{i+1}^{*}, \ldots, a_{n}^{*}\right) \\
a_{i+1}^{*}=s_{i+1}\left(h_{i+1}, r\right), h_{i+1}=\left(h_{i}, a_{i}\right) \\
\forall j \in\{i+2, \ldots, n\}\left\{a_{j}^{*}=s_{j}\left(h_{j}, r\right), h_{j}=\left(h_{j-1}, a_{j-1}^{*}\right)\right\} \\
\forall \tilde{\jmath} \in N\left\{a_{\tilde{\jmath}}^{*}=s_{\tilde{\jmath}}\left(h_{\tilde{\jmath}}, r+1\right), h_{\tilde{\jmath}}=\left(h_{\tilde{\jmath}-1}, a_{\tilde{\jmath}-1}^{*}\right)\right\} .
\end{gathered}
$$

In any state of any round $r$, when it is bidder $i$ 's turn to bid, he uses the above optimal function to calculate the optimal $\mathrm{bid} /$ response, given the previous bids in the current round $r$.

We let $S_{i}^{r}$ denote the equilibrium strategy of a player when he is represented by bidder $i \in N$ in round $r \leq \gamma$, let $S^{r}$ denote the equilibrium strategies in round $r$ where $S^{r}=$ $\left(S_{1}^{r}, \ldots, S_{n}^{r}\right)$, and let $S=\left(S^{1}, \ldots, S^{\gamma}\right)$ denote the strategy profile of the players of this game.

Proposition 1. The equilibrium strategy of bidder $i \in N$ in round $r \leq \gamma$ is $S_{i}^{r}$, which is given by Algorithm 1. The strategy profile $S=\left(S^{1}, \ldots, S^{\gamma}\right)$ induces a subgame perfect equilibrium of the game. If an agreement exists in this game, it will be reached immediately at the end of round 1.

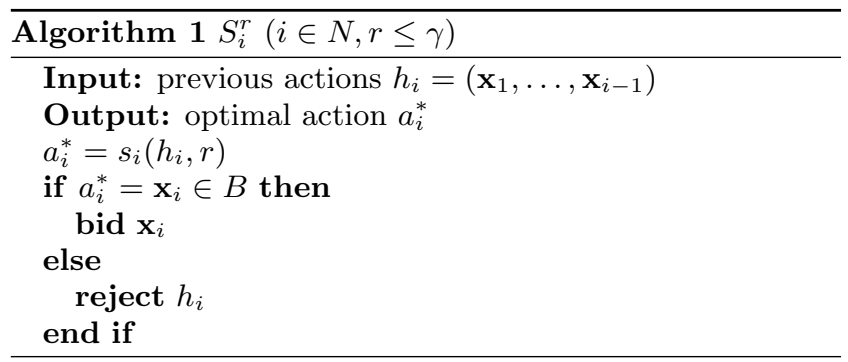

Proof. We argue that the strategy profile $S$ forms a subgame perfect equilibrium, so we need to show that $S$ induces a Nash equilibrium in every subgame (round). We are going to prove that the optimal action of any bidder $i \in N$ in any round $r \leq \gamma$ given by the equilibrium strategy $S_{i}^{r}$ is the best response to the optimal actions of his opponents $-i$ given by the equilibrium strategies $S_{-i}^{r}$. We give a proof by contradiction.

In any round $r$, when it is bidder $i$ 's turn to bid, his optimal action is $a_{i}^{*}=S_{i}^{r}$, given the previous bids induced by the equilibrium strategies. We let $a_{-i}^{*}$ denote action profile induced by the equilibrium strategies $S_{-i}^{r}$, given $a_{i}^{*}$. Suppose any other strategy used by bidder $i$ is to choose another action $a_{i}^{\prime} \in A$ where $u_{i}\left(\left(a_{i}^{\prime}, a_{-i}^{\prime}\right), r\right)>u_{i}\left(\left(a_{i}^{*}, a_{-i}^{*}\right), r\right)$, when all players other than $i$ adhere to $S_{-i}^{r}$ which induces the action profile $a_{-i}^{\prime}$, given $a_{i}^{\prime}$.

When the profile of optimal actions $a^{*}=\left(a_{i}^{*}, a_{-i}^{*}\right)$ can form an agreement in round $r$, the utility $u_{i}\left(a^{*}, r\right)$ has been maximized in Equation (2) while the utility $u_{j}\left(a^{*}, r\right)$ is no less than the utility that bidder $j$ would get in round $r+1$ where $j \in N$. Because all utility functions are monotonically increasing, if the action profile $a^{\prime}=\left(a_{i}^{\prime}, a_{-i}^{\prime}\right)$ is a profile of bids and increases the utility for bidder $i, a^{\prime}$ either violates the constraint $(\mathcal{C})$ or lets at least one of other bidders get a utility less than the utility that he would get in round $r+1$, if $a^{\prime}$ is really implemented. Hence, the action profile $a^{\prime}$ cannot form an agreement in round $r$, which is the same as the situation that reject $\in a^{\prime}$. In both situations, the utility that bidder $i$ can get by taking action $a_{i}^{\prime}$ in round $r$ equals to the utility that he would get in round $r+1$, which is no more than the utility $u_{i}\left(a^{*}, r\right)$. There is a contradiction. Because the utility function defined in this work is completely general, it is possible that no agreement exists in the game. For any action profile, every player gets the same utility, zero, so there is also a contradiction. Therefore, the optimal action $a_{i}^{*}$ is bidder $i$ 's best action/response to his opponents' actions induced by the equilibrium strategies. The equilibrium strategies $S^{r}$ induces a Nash equilibrium in round $r$ and the strategy profile $S$ induces Nash equilibrium in every round $r \leq \gamma$, which is a subgame perfect equilibrium.

When the strategy profile $S$ can form an agreement $\mathbf{x}$ in round $r \leq \gamma$, then every bidder $i \in N$ gets a utility $u_{i}(\mathbf{x}, r)$, which is no less than the utility that he would get in the next round $r+1$. The bid profile $\mathbf{x}$ can also be an agreement in round $r+1$. Because of the discount factor $\delta$, at least one bidder $i$ in round $r$ has $u_{i}(\mathbf{x}, r)>u_{\tilde{\imath}}(\mathbf{x}, r+1)$ and any other bidder $j$ in round $r$ has $u_{j}(\mathbf{x}, r) \geq u_{\tilde{\jmath}}(\mathbf{x}, r+1)$. Under the assumption of benevolence, an agreement reached earlier is always preferred by all players. Thus, an agreement will be reached immediately at the end of round 1 and the negotiation stops.

In the optimal action function (2), it is possible that bidder $i \in N$ has multiple bids that have the same maximum utility, which are indifference to bidder $i$ but not to bidder $j>i$. There may be an opportunity to increase the outcome utility for bidder $j$ without decreasing the outcome utility for bidder $i$. Therefore, under the assumption of benevolence, if bidder $i$ has multiple bids that have the same maximum utility, we let bidder $i$ choose the one that is best for bidder $i+1$. If bidder $i$ still has more than one bid that is best for bidder $i+1$, we let bidder $i$ choose the one that is best for bidder $i+2$, etc. This selection will last until bidder $i$ has only one optimal bid left or bidder $n$ has already 
been considered by bidder $i$. We call this as the completely benevolent selection.

Proposition 2. The equilibrium outcome is a Pareto optimal solution of the game if every player chooses his optimal action with the completely benevolent selection.

Proof. We argue that the equilibrium outcome is Pareto optimal, so we need to prove that no other outcome can increase the outcome utility for any player without decreasing the outcome utilities for any other player, when every player chooses his optimal action with the completely benevolent selection. We give proof by contradiction.

When the equilibrium strategies $S$ can reach an agreement $\mathbf{x}$ at the end of round 1. Suppose bidder $i \in N$ has another bid $\mathbf{x}_{i}^{\prime}$ that the bid profile $\mathbf{x}^{\prime}=\left(\mathbf{x}_{i}^{\prime}, \mathbf{x}_{-i}^{\prime}\right)$ also forms an agreement in that round where $u_{i}\left(\mathbf{x}^{\prime}, 1\right)>u_{i}(\mathbf{x}, 1)$ and $u_{j}\left(\mathbf{x}^{\prime}, 1\right) \geq u_{j}(\mathbf{x}, 1)(j \in N-i)$. Because all utility functions are monotonically increasing and $u_{i}(\mathbf{x}, 1)$ has been maximized in Equation (2) with the completely benevolent selection, the bid profile $\mathbf{x}^{\prime}$ either violates the constraint $(\mathcal{C})$ so that $\mathbf{x}^{\prime}$ is not an agreement or lets at least one bidder $j$ get $u_{j}\left(\mathbf{x}^{\prime}, 1\right)<u_{j}(\mathbf{x}, 1)$. There is a contradiction. Therefore, the equilibrium outcome $\mathbf{x}$ is a Pareto optimal.

When no agreement can be reached by using the equilibrium strategies $S$, every player gets zero utility. It is impossible to increase the utility of any player without rejections from other players Therefore, the equilibrium outcome introduced by $S$ is a Pareto optimal solution of the game.

Proposition 3. The negotiation mechanism is individually rational.

Proof. Because whether the outcome is an agreement or disagreement, every player gets a utility no less than zero, which is also the utility for every player if he does not participate the game, the negotiation mechanism is individually rational.

\subsection{A Simple Example}

In this section, we use a simple example to illustrate the negotiation model and equilibrium strategies. Suppose three students need to share an office. They all prefer to have the office only to themselves. They therefore decide to timeshare the office, but they agree to allow the others to leave their stuff (books, etc.) behind in the cupboard. Each of the students would like to have the office as long as possible. Let therefore the first issue be the part of the working day a student has on his own. They also like to get as much space in the cupboard as possible. Let the second issue thus be the part of the cupboard they are entitled to. The dean overheard them discussing and said: "you should take turns in making proposals to each other, but if you haven't reached an agreement before noon, I'll give the room to someone else. Determine the order of the terms by drawing straws."

Given the above case, we let three players, 1,2 and 3, denote the students and let the shares of time and cupboard be the first issue and the second issue respectively. We let $x_{i, k}$ denote the $i^{\text {th }}$ player's proposal for the $k^{t h}$ issue and let $x_{i, k} \in\{0.1, \ldots, 0.9\}$ for simplicity reasons where $i=1,2,3$ and $k=1,2$. We assume that there are at most three negotiation rounds and the bidding orders are $\langle(1,2,3),(2,3,1),(3,1,2), \ldots\rangle$ given in Section 2 . We define the following valuation functions for an agreement $\mathbf{x}=$ $\left(\mathbf{x}_{1}, \mathbf{x}_{2}, \mathbf{x}_{3}\right)$ for each of the players:

$$
v_{1}\left(\mathbf{x}_{1}\right)=8 \times x_{1,1}+2 \times x_{1,2}-1.5
$$

Table 1: Example of 3-player 2-issue Negotiation

\begin{tabular}{|c||c|c|}
\hline round 1 & $\mathbf{x}_{i}^{1}$ & $u_{i}^{1}$ \\
\hline$i=1$ & $(0.2,0.1)$ & $0.3>0.24$ \\
\hline$i=2$ & $(0.7,0.2)$ & $3.2>2.16$ \\
\hline$i=3$ & $(0.1,0.7)$ & $3.8>3.6$ \\
\hline \hline round 2 & $\mathbf{x}_{i}^{2}$ & $u_{i}^{2}$ \\
\hline$i=2$ & $(0.7,0.1)$ & $2.16>0.128$ \\
\hline$i=3$ & $(0.1,0.8)$ & $3.6>3.584$ \\
\hline$i=1$ & $(0.2,0.1)$ & $0.24>0.192$ \\
\hline \hline round $\gamma$ & $\mathbf{x}_{i}^{\gamma}$ & $u_{i}^{\gamma}$ \\
\hline$i=3$ & $(0.7,0.7)$ & $3.584>0$ \\
\hline$i=1$ & $(0.2,0.1)$ & $0.192>0$ \\
\hline$i=2$ & $(0.1,0.2)$ & $0.128>0$ \\
\hline
\end{tabular}

$$
\begin{aligned}
& v_{2}\left(\mathbf{x}_{2}\right)=5 \times x_{2,1}+5 \times x_{2,2}-1.3 \\
& v_{3}\left(\mathbf{x}_{3}\right)=3 \times x_{3,1}+7 \times x_{3,2}-1.4
\end{aligned}
$$

These valuation functions are part of the utility function of each player $i$, as defined in Equation (1). We set the discount factor to $\delta=0.8$. The optimal bid of every player in every round according to the equilibrium strategy is given in Table 1 below. This table shows that in each round, the three bids form an agreement, and that every player's utility is at least as high as his utility in the next round. Unless one or more players submit bids other than their equilibrium strategies, the negotiation will stop at the end of the first round.

As a final example, suppose that player 3 bids $(0.8,0.8)$ in the last round, deviating from its equilibrium strategy. In that case, either player 1 or player 2 will not receive enough of the issues to obtain a positive utility. Therefore, no agreement will be reached and every player will get zero utility.

\section{RELATED WORK}

In this section, we discuss some related work of multiplayer and/or multi-issue negotiation with complete information. The best known negotiation model is the alternatingoffer bargaining game [14]. Basically, in a two-player game, one player proposes a partition of a single issue to the other player. The opponent can accept the proposal or make a counter-proposal or quit the negotiation. The negotiation continues until reaching an agreement or a finite deadline. Ståhl identifies the optimal strategies for rational players with perfect information in such a game with a finite time horizon [17]. Rubinstein identifies a unique SPE, which is reached immediately, in a perfect information setting with an infinite time horizon [14]. The Ståhl-Rubinstein model [14], two-player single-issue bargaining, has been extended into two directions, either the negotiation between more than two players or the multi-issue negotiation. The model of $n$-player single-issue negotiation has been investigated in $[2,9,16]$. One proposer is chosen by a pre-specified order in each stage of a multi-stage game; he proposes a partition of one issue for all players and other players then respond sequentially. If players have time preferences with a common constant discount factor, there is a unique allocation of a pie amongst three players, which tends to an equal partition as players become more patient [9]. It is possible to obtain an equilibrium similar to the unique SPE of the two-player game by limiting the strategies available to players to his- 
tory independent strategies [16]. Some other work addresses the multiple players game by modifying the structure of the game. For instance, players are engaged in a series of bilateral negotiation [8] and any player that reaches a satisfactory agreement may "exit" the game $[4,11]$. Two-player multiissue negotiation has been studied in two ways. Multiple issues are negotiated one by one, so the role of a negotiation agenda has been studied by various work $[1,3,5,7,10]$. Alternatively, multiple issues can be treated as one package. A comparison between the package approach and the sequential approach is made in [6] and the former shows a better outcome as it introduces the opportunity of trade-offs.

The model built in this work includes both many players and many issues. We let each player bid only for himself sequentially; every bid is searched in an inherently infinite set of bids. The game is multilateral and all issues are negotiated as one package. Both a common deadline and a common constant discount factor are set; players are not permitted to exit. A model of many player and multidimensional issue spaces has been studied in [13]. In that work, according to a pre-specified vector of "access probabilities", one proposer is selected in each negotiation round. The solution is a limit of equilibrium outcomes, as the number of negotiation rounds increases without bound. Their model let $n$ players form multiple admissible coalitions. If an admissible coalition has the proposer and his proposal is accepted by all members in that coalition, the proposal will be imposed to all $n$ players. The model is more practical, especially in political field. Compared to it, our model is more general and can be directly used on $n$ equal players.

\section{CONCLUSIONS AND FUTURE WORK}

In this paper, we have proposed a general multi-player multi-issue multilateral negotiation protocol. Given two time constraints, the deadline and the discount factor, we proposed equilibrium strategies under a complete information setting. Given these strategies, an agreement can be reached immediately at the end of the first round, if it exists, and the solution is a subgame perfect equilibrium. By making tradeoffs between issues, every player's utility in the equilibrium outcome is maximized and the solution is Pareto optimal. To our knowledge, this is one of the first papers to study, from a game theoretic perspective, the case of multi-issue negotiation with multiple players. This case introduces a new level of complexity to deriving subgame perfect equilibrium strategies, in comparison to bilateral bargaining. The result of this work can be widely and directly used to solve allocation problems of resources, tasks, etc.

With the technique developed in this paper, we are currently developing a solution for the incomplete information cases, in which the optimal actions of players are concerned with their beliefs about types of each other. This is a complex problem as those beliefs will change due to ongoing new bidding information. In our work, we not only update players' beliefs during the real negotiation (similar to earlier work on (bilateral) negotiation with incomplete information [6]), but also take such updates into account when the players reason about their optimal actions.

Besides the incomplete information case, there are several other interesting directions for extending this work. It will be interesting to study a model where different players have different deadlines and discount factors also. If the bidding order of each round cannot be determined before the ne- gotiation, the equilibrium strategies will be quite different. Finally, we can try relaxing the constraint of monotonicity and study the model with more general utility functions.

\section{REFERENCES}

[1] M. Bac and H. Raff. Issue-by-Issue Negotiations: The Role of Information and Time Preference. Games and Economic Behavior, 13(1):125-134, 1996.

[2] K. Binmore. Bargaining and Coalitions. Game-Theoretic Models of Bargaining, pages 269-304, 1985.

[3] L. Busch and I. Horstmann. Bargaining Frictions, Bargaining Procedures and Implied Costs in Multiple-Issue Bargaining. Economica, 64(256):669-680, 1997.

[4] S. Chae and J. Yang. An N-person pure bargaining game. Journal of Economic Theory, 62(1):86-102, 1994.

[5] S. Fatima, M. Wooldridge, and N. Jennings. An agenda-based framework for multi-issue negotiation Artificial Intelligence, 152(1):1-45, 2004.

[6] S. Fatima, M. Wooldridge, and N. Jennings. Multi-Issue Negotiation with Deadlines. Journal of Artificial Intelligence Research, 27:381-417, 2006.

[7] C. Fershtman. The importance of the agenda in bargaining. Games and Economic Behavior, 2(3):224-238, 1990 .

[8] E. Gerding, D. Somefun, and J. La Poutre. Multi-attribute Bilateral Bargaining in a One-to-Many Setting. LECTURE NOTES IN COMPUTER SCIENCE, 3435:129, 2005.

[9] M. Herrero. A Strategic Bargaining Approach to Market Institutions. Unpublished Ph. D. Thesis. University of London, 1985.

[10] R. Inderst. Multi-issue Bargaining with Endogenous Agenda. Games and Economic Behavior, 30(1):64-82, 2000.

[11] B. Jun. A Structural Consideration on 3-Person Bargaining. Chapter III in Essays on Topics in Economic Theory, Ph. D. Thesis, Department of Economics, University of Pennsylvania, 1987.

[12] M. Osborne. An introduction to game theory. Oxford University Press New York, 2004.

[13] G. Rausser and L. Simon. A non-cooperative model of collective decision making: A multilateral bargaining approach. Department of Agricultural and Resource Economics (University of California, Berkeley), 1992.

[14] A. Rubinstein. Perfect Equilibrium in a Bargaining Model. Econometrica, 50(1):97-110, 1982.

[15] R. Selten. Spieltheoretische Behandlung eines Oligopolmodells mit Nachfragetragheit. Zeitschrift fur die gesamte Staatswissenschaft, 12:301-324, 1965.

[16] A. Shaked. A Three-Person Unanimity Game. Talk given at the Los Angeles national meetings of the Institute of Management Sciences and the Operations Research Society of America, 1986.

[17] I. Ståhl. Bargaining theory. (Ekonomiska forskningsinstitutet vid Handelshögskolan i Stockholm (EFI)), 1972. 